\title{
Systemic versus Oral and Systemic Antibiotic Prophylaxis (SOAP) study in colorectal surgery: prospective randomized multicentre trial
}

\author{
G. Papp (DD ${ }^{1, *}$, Gy. Saftics ${ }^{1}$, B. E. Szabó ${ }^{1}$, J. Baracs² ${ }^{2}$ A. Vereczkei² , D. Kollár ${ }^{3}$, A. Oláh ${ }^{3}$, P. Mészáros ${ }^{4}$, Zs. Dubóczki and A. Bursics ${ }^{1}$ \\ ${ }^{1}$ Department of General Surgery and Surgical Oncology, Uzsoki Hospital, Budapest, Hungary \\ ${ }^{2}$ Department of Surgery, University of Pécs Clinical Centre, Pécs, Hungary \\ ${ }^{3}$ Department of General Surgery, Petz Aladár Teaching Hospital, Györ, Hungary \\ ${ }^{4}$ Department of Visceral Surgery, National Institute of Oncology, Centre of Oncosurgery, Budapest, Hungary \\ *Correspondence to: Department of General Surgery and Surgical Oncology, Uzsoki Hospital, Róna u. 196-212, Budapest, 1145 Hungary (e-mail: papp.geza@uzsoki.hu)
}

\begin{abstract}
Background: There is no consensus regarding the role of mechanical bowel preparation (MBP) and oral antibiotic prophylaxis (OABP) in reducing postoperative complications in colorectal surgery. The aim of this study was to examine the effect of OABP given in addition to MBP in the setting of a prospective randomized trial.

Methods: Patients awaiting elective colorectal surgery in four Hungarian colorectal centres were included in this multicentre, prospective, randomized, assessor-blinded study. Patients were randomized to receive MBP with or without OABP (OABP+ and OABP- groups respectively). The primary endpoints were surgical-site infection (SSI) and postoperative ileus. Secondary endpoints were anastomotic leak, mortality, and hospital readmission within 30 days.

Results: Of 839 patients assessed for eligibility between November 2016 and June 2018, 600 were randomized and 529 were analysed. Trial participation was discontinued owing to adverse events in seven patients in the OABP + group (2.3 per cent). SSI occurred in eight patients ( 3.2 per cent) in the OABP + and 27 (9.8 per cent) in the OABP- group $(P=0.001)$. The incidence of postoperative ileus did not differ between groups. Anastomotic leakage occurred in four patients (1.6 per cent) in the OABP+ and 13 (4.7 per cent) in the OABP- $(P=0.02)$ group. There were no differences in hospital readmission (12 (4.7 per cent) versus 10 (3.6 per cent); $P=0.25)$ or mortality (3 (1.2 per cent) versus 4 (1.4 per cent); $P=0.39$ ).
\end{abstract}

Conclusion: OABP given with MBP reduced the rate of SSI and AL after colorectal surgery with anastomosis, therefore routine use of OABP is recommended.

\section{Introduction}

Although intravenous antibiotic prophylaxis is used routinely for colorectal surgery ${ }^{1}$, the role of preoperative oral antibiotic prophylaxis $(\mathrm{OABP})^{2}$ and mechanical bowel preparation $(\mathrm{MBP})^{3}$ is more controversial, despite a Cochrane review that supported the combined use of oral and intravenous antibiotics to reduce surgical-site infection (SSI) .

The combination of OABP and MBP is used widely in North America, and several studies ${ }^{5-8}$ have supported its use. Recent guidelines from the American Society of Colon and Rectal Surgeons ${ }^{9}$ recommend the use of both MBP and OABP, a practice also endorsed by the American Society of Enhanced Recovery ${ }^{10}$. Data from several thousand patients in the American College of Surgeons' National Surgical Quality Improvement Program have shown that OABP given with MBP (and intravenous antibiotic prophylaxis) reduces SSI, anastomotic insufficiency, postoperative ileus, 30-day mortality, and 30-day readmission rates ${ }^{7,11-13}$. Several expert panels ${ }^{9,14}$ and review studies ${ }^{15-19}$ have addressed the use of $\mathrm{OABP}$, but relatively few prospective studies ${ }^{20-28}$ have reported to date. The majority of meta-analyses concluded that OABP in combination with MBP reduces SSI after colorectal surgery $^{16-19,22,29}$.

To date, European data have been conflicting. A nonrandomized, multicentre audit ${ }^{30}$ found significantly less anastomotic insufficiency after left-sided colorectal resection if the patients were given OABP in combination with MBP. However, a recent prospective, randomized trial ${ }^{31}$ reported no benefit of $\mathrm{OABP}$ in combination with MBP compared with a no-preparation (NoPrep) policy. The results of some ongoing prospective trials are yet to be published ${ }^{32,33}$.

The aim of the SOAP (Systemic versus Oral and systemic Antibiotic Prophylaxis) trial was to examine the effects of OABP given in combination with MBP and intravenous antibiotic prophylaxis. The hypothesis was that patients who received MBP, intravenous antibiotic prophylaxis and OABP (OABP+ group) would have improved outcomes compared with those who received 
MBP and intravenous antibiotic prophylaxis only (OABP- group). The primary endpoints were rates of SSI and postoperative ileus, and secondary endpoints were anastomotic leak rate, mortality, and hospital readmission within 30 days.

\section{Methods}

The SOAP study was non-commercial, multicentre, prospective, randomized, superiority, assessor-blinded study registered as EudraCT 2015-005614-30. Ethical approval was granted by both the Hungarian National Institute of Pharmacy and Nutrition and the Hungarian Medical Research Council.

All patients gave informed consent to participate in the study. All indications for colorectal anastomosis were considered eligible, including Hartmann's reversal, with the exception of loop colostomy closure. Patients were classified into left- and right-sided groups according to the site of resection and anastomosis. If both sides of the colon were removed (colectomy/double resection), the procedure was categorized as a left-sided resection. Anastomosis to the lower two-thirds of the rectum was considered a rectal anastomosis. Patients were excluded if they had received antibiotics within 2 weeks before randomization, were allergic to any of the drugs used, were aged less than 18 years, had abdominal sepsis within 6 months before randomization, were pregnant or breast feeding, were being treated with steroids or had any form of chronic immunosuppression, or had obstructive symptoms. Patients were excluded after randomization if they did not receive the study drugs according to the study protocol or if they did not have an anastomosis created during surgery for any reason.

All patients received bowel preparation comprising $40 \mathrm{ml}$ castor oil with $20 \mathrm{ml}$ paraffin on the day before surgery. An enema was given on the evening before surgery and again on the morning of surgery. All patients received intravenous $2 \mathrm{~g}$ ceftriaxone and $500 \mathrm{mg}$ metronidazole within $60 \mathrm{~min}$ of the incision. This was repeated if operating time exceeded $4 \mathrm{~h}$ and/or blood loss exceeded $1500 \mathrm{ml}$. Patients in the investigation arm (OABP+) were given $500 \mathrm{mg}$ metronidazole and $1000 \mathrm{mg}$ neomycin sulphate orally at $13.00,15.00$, and 19.00 hours on the day before surgery.

Patients were followed until postoperative day 30, in accordance with recommendations from the Centers for Disease Control and Prevention ${ }^{34}$. Any wound discharge was considered to represent at least a superficial wound infection. If the deeper layers (fascia, musculature) were affected, it was classified as deep SSI. When infection manifested within the abdominal cavity (any fluid collection, abscess), it was regarded as an organ space (abdominal) SSI. Imaging was performed only when indicated clinically. Postoperative ileus was defined by the need for a nasogastric tube and/or the patient being nil by mouth on day 3 or more after operation. Any clinically or radiologically proven anastomotic suture dehiscence was counted as an anastomotic leak. If anastomotic leakage was proven, the patient was not included in the organ space SSI group for analysis. During surgery, the surgeon evaluated the success of MBP according to the Boston Bowel Preparation Scale (BBPS) ${ }^{35}$. MBP was considered successful if the BBPS score was at least 2. Four high-volume colorectal Hungarian centres participated in the study; the procedures were performed by 21 surgeons. All centres used the same colorectal care bundle and enhanced recovery after surgery (ERAS) protocol.

\section{Statistical analysis}

The study power calculation was based on the international literature $^{7,11,12}$, with an estimated 11 per cent incidence of SSI in the $\mathrm{OABP}$ - group and 5 per cent in the OABP+ group. Postoperative ileus was estimated to occur in 6 per cent of patients in the $\mathrm{OABP}-$ group and 3 per cent in the OABP+ group. Using $\delta=3$ and an adjusted study power of 80 per cent with a 95 per cent confidence interval, it was calculated that 282 patients were required for the SSI primary endpoint and 374 for the postoperative ileus endpoint. This was rounded up to 400 patients and, after adjusting for a possible 12.5 per cent loss, the final sample size was estimated to be 450 patients. Data were recorded in the Research Electronic Data Capture (REDCap) system, which was also used for randomization. Randomization was stratified by age. The assessors were blinded to the assigned intervention arm. R statistical software (R Foundation for Statistical Computing, Vienna, Austria) was used for data analysis. Variables were compared using the $\mathrm{z}$ test (with 1 - or 2 -sided confidence interval), $\chi^{2}$ test, ANOVA or multivariable linear regression, as appropriate. A 5 per cent significance level was accepted.

\section{Results}

In the four participating centres, 839 patients undergoing elective colorectal surgery with a planned anastomosis were assessed for eligibility between November 2016 and June 2018, of whom 600 were randomized. Of these 600 patients, 71 patients were excluded either because of a protocol violation or because an anastomosis was not created, leaving 529 patients for analysis (Fig. 1). The breakdown of operations was 181 (34.2 per cent) involving the right colon, 167 (31.6 per cent) the left colon, and 181 (34.2 per cent) the rectum.

Indications for surgery were tumour (malignant or benign) in 461 patients (87.1 per cent), inflammatory bowel or diverticular disease in 33 (6.2 per cent), reversal of Hartmann's procedure in 30 (5.7 per cent), and other (angiodysplasia or functional bowel problems) in five patients ( 0.9 per cent). Patient characteristics of the study groups are summarized in Table 1.

Adverse events related to oral antibiotic use were reported by 32 patients: nausea or vomiting (29), abdominal cramps (2), dizziness (2), and numbness of fingers or toes (3); some patients experienced more than one adverse event. None required intervention. Seven of the 32 patients decided to withdraw from the study owing to side-effects.

The results for primary and secondary endpoints are shown in Table 2. There was an overall reduction in SSI in the OABP+ group $(P=0.001)$, particularly in superficial SSIs $(P=0.01)$. For organ space SSI, the difference approached significance $(P=0.06)$ in favour of the OABP+ group. There was no difference in deep SSI between the study groups. The rate of postoperative ileus did not differ between groups. Regarding secondary endpoints, anastomotic leakage occurred less frequently in the OABP+ group, whereas there was no difference in 30-day mortality and readmission rates.

The results of multivariable linear regression analysis are shown in Table 3. The addition of OABP independently reduced rates of both SSI and anastomotic leak $(P<0.001$ and $P=0.048$ respectively). Laparoscopic surgical access also independently reduced SSI $(P=0.003)$ and 30-day readmission $(P=0.03)$ rates compared with open surgery. Rectal anastomosis was associated with a higher risk of anastomotic leak $(P=0.001)$ and 30-day 

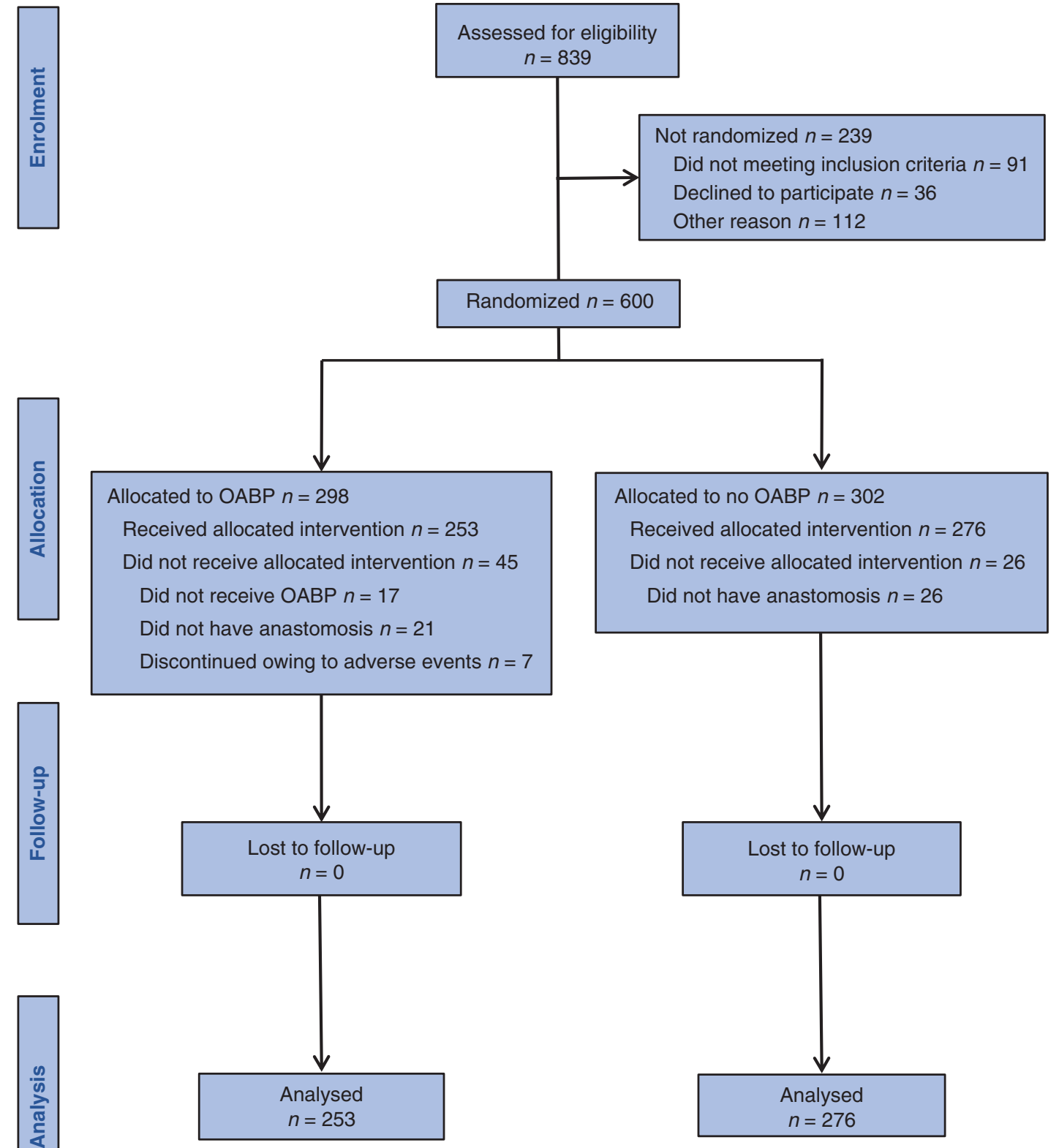

Fig. 1 CONSORT flow chart for SOAP study

OABP, oral antibiotic prophylaxis.

mortality $(P=0.03)$. Subgroup analyses of the impact of the success of MBP on SSI and anastomotic leak rates are provided in Table 4.

\section{Discussion}

In this study, OABP used with MBP reduced postoperative SSI after elective colorectal resection involving an anastomosis, regardless of the success of MBP. This effect was most marked for the rate of superficial SSI, but was also observed in deep and organ space SSI. It was also found that OABP used with MBP reduced the incidence of anastomotic leak. Subgroup analysis suggested that adequate MBP (BBPS score 2 or more) is important to reduce anastomotic leakage and also the adequate MBP without OABP reduced SSI.

The two primary endpoints of this study were rates of SSI and postoperative ileus. Although SSI rates were reduced, no difference was seen in the incidence of postoperative ileus. This might be related to the lower than anticipated overall incidence of postoperative ileus in the study, which could be explained by the routine use of ERAS protocols in all four participating institutes.

The role of OABP before elective colorectal surgery is controversial; previous underpowered studies have demonstrated variable results. A single-centre study by Espin-Basany and colleagues ${ }^{23}$ compared three groups: MBP and three doses of OABP, $\mathrm{MBP}$ and one dose of OABP, and no OABP. All patients received intravenous second-generation cephalosporin prophylaxis. It was 
Table 1 Patient characteristics

Oral antibiotic prophylaxis $(n=253)$

No oral antibiotic prophylaxis $(n=276)$

\begin{tabular}{lcc}
\hline Age (years) & $66.5(12.3)$ \\
BMI $\left(\mathrm{kg} / \mathrm{m}^{2}\right)^{*}$ & $66.1(12.1)$ & $27.3(4.5)$ \\
Sex ratio $(\mathrm{F}: \mathrm{M})$ & $27.0(4.3)$ & $146: 130$ \\
Cardiac disease & $101: 152$ & $85(30.8)$ \\
Diabetes mellitus & $101(39.9)$ & $50(18.1)$ \\
COPD & $44(17.4)$ & $19(6.9)$ \\
Anticoagulant therapy & $34(13.4)$ & $37(13.4)$ \\
Tumour category & $44(17.4)$ & $13(4.7)$ \\
T1 & & $51(18.5)$ \\
T2 & $18(7.1)$ & $114(41.3)$ \\
T3 & $43(17.0)$ & $29(10.5)$ \\
T4 & $98(38.7)$ & $69(25.0)$ \\
Not malignant or tumour unknown & $23(9.0)$ & $169(61.2)$ \\
BBPS score $\geq 2$ & $71(28.1)$ & $157(56.9)$ \\
Laparoscopic procedure & $159(62.8)$ & $90(32.6)$ \\
Rectal anastomosis & $134(53.0)$ & 42 of $81(52)$ \\
Neoadjuvant therapy for rectal cancer & $91(36.0)$ & (5)
\end{tabular}

Values in parentheses are percentages unless indicated otherwise; "values are mean(s.d.). COPD, chronic obstructive pulmonary disease; BBPS, Boston Bowel Preparation Scale.

Table 2 Intraoperative and postoperative complications

\begin{tabular}{|c|c|c|c|c|}
\hline & $\begin{array}{l}\text { Oral antibiotic prophylaxis } \\
\qquad(n=253)\end{array}$ & $\begin{array}{l}\text { No oral antibiotic prophylaxis } \\
\qquad(n=276)\end{array}$ & $z$ & $P^{*}$ \\
\hline $\begin{array}{l}\text { Intraoperative anaesthestic complication } \\
\text { Complications by Clavien-Dindo grade }\end{array}$ & $17(6.7)$ & $11(4.0)$ & 1.40 & $\begin{array}{l}0.081 \\
0.689^{\dagger}\end{array}$ \\
\hline I & $41(16.2)$ & $42(15.2)$ & & \\
\hline II & $31(12.3)$ & $44(15.9)$ & & \\
\hline IIIa & $2(0.8)$ & $1(0.4)$ & & \\
\hline $\mathrm{IVb}$ & $1(0.4)$ & $0(0)$ & & \\
\hline V & $3(1.2)$ & $4(1.4)$ & & \\
\hline Clostridium difficile infection & $4(1.6)$ & $2(0.7)$ & 0.92 & 0.176 \\
\hline Overall SSI & $8(3.2)$ & $27(9.8)$ & -3.06 & 0.001 \\
\hline Superficial SSI & $6(2.4)$ & $18(6.5)$ & -2.29 & 0.011 \\
\hline Deep SSI & $1(0.4)$ & $4(1.4)$ & -1.25 & 0.105 \\
\hline Organ space SSI & $1(0.4)$ & $5(1.8)$ & -1.53 & 0.061 \\
\hline
\end{tabular}

Values in parentheses are percentages. SSI, surgical-site infection. ${ }^{*} \mathrm{z}$ test, except ${ }^{\dagger} \chi^{2}$ test.

Table 3 P values from multivariable linear regression analysis of the effect of demographic and clinical variables on primary and secondary endpoints

\begin{tabular}{|c|c|c|c|c|c|}
\hline & \multicolumn{5}{|c|}{$P$} \\
\hline & SSI & Postoperative ileus & Anastomotic leakage & Mortality & Hospital readmission \\
\hline Age > mean (66.3 years) & 0.437 & 0.464 & 0.127 & 0.170 & 0.287 \\
\hline $\mathrm{BMI}>\operatorname{mean}\left(27.2 \mathrm{~kg} / \mathrm{m}^{2}\right)$ & 0.391 & 0.391 & 0.174 & 0.307 & 0.421 \\
\hline Male sex & 0.249 & 0.005 & 0.479 & 0.171 & 0.083 \\
\hline Cardiac disease & 0.357 & 0.246 & 0.061 & 0.334 & 0.447 \\
\hline Diabetes mellitus & 0.464 & 0.464 & 0.323 & 0.402 & 0.297 \\
\hline COPD & 0.406 & 0.040 & 0.083 & 0.348 & 0.020 \\
\hline Anticoagulant therapy & 0.083 & 0.153 & 0.347 & 0.446 & 0.161 \\
\hline Oral antibiotic prophylaxis & $<0.001$ & 0.332 & 0.048 & 0.392 & 0.256 \\
\hline BBPS score $\geq 2$ & 0.052 & 0.190 & 0.481 & 0.106 & 0.242 \\
\hline Laparoscopic surgical access & 0.003 & 0.121 & 0.337 & 0.252 & 0.031 \\
\hline Rectal anastomosis & 0.118 & 0.207 & 0.001 & 0.031 & 0.232 \\
\hline Neoadjuvant therapy & 0.461 & 0.216 & 0.208 & 0.133 & 0.116 \\
\hline Intraoperative surgical complication & 0.164 & 0.336 & 0.404 & 0.024 & 0.581 \\
\hline Intraoperative anaesthestic complication & 0.093 & 0.321 & 0.173 & 0.002 & 0.203 \\
\hline
\end{tabular}

SSI, surgical-site infection; COPD, chronic obstructive pulmomary disease; BBPS, Boston Bowel Preparation Scale. 
Table 4 Subgroup analyses of impact of success of mechanical bowel preparation on surgical-site infection and anastomotic leak rates

\begin{tabular}{|c|c|c|c|c|c|}
\hline & No. of patients & $\begin{array}{c}\text { BBPS score } \geq 2 \\
(n=328)\end{array}$ & $\begin{array}{c}\text { BBPS score } \leq 1 \\
(n=129)\end{array}$ & $z$ & $\mathrm{P}^{*}$ \\
\hline \multicolumn{6}{|l|}{ Surgical-site infection } \\
\hline No oral antibiotic prophylaxis & 240 & 14 of $169(8.3)$ & 11 of $71(15)$ & -1.67 & 0.047 \\
\hline z & & -1.99 & -1.87 & & \\
\hline$P^{*}$ & & 0.023 & 0.031 & & \\
\hline \multicolumn{6}{|l|}{ Anastomotic leak } \\
\hline $\mathrm{z}$ & & -2.66 & -0.23 & & \\
\hline$P^{*}$ & & 0.004 & 0.409 & & \\
\hline
\end{tabular}

Values in parentheses are percentages. BBPS, Boston Bowel Preparation Scale. "z test.

found that OABP did not influence SSI rates, but gastrointestinal side-effects were experienced by 40 per cent of patients who received $\mathrm{OABP}$. In the present study, the side-effects of OABP were mild, and only 2.3 per cent of patients stopped the medication because of side-effects.

The recent MOBILE trial ${ }^{31}$ compared patients who received MBP and OABP with those treated according to a NoPrep policy. Although there was a 50 per cent increase in complications in the NoPrep group (11 versus 7 per cent), the authors concluded that there was no significant difference between the two groups. This was most probably because the study was underpowered to detect a 4 per cent difference.

The present study has some weaknesses. It was not doubleblinded; however, double-blinding might have hindered patient recruitment. Radiological screening for anastomotic leak was not undertaken routinely as this was not normal practice at any of the participating sites. The strength of the study is that it is a prospective, randomized, assessor-blinded study with wide inclusion criteria. Patients were not selected according to indications, procedure types or surgical approach (open/laparoscopic). Therefore, the study groups reflect current routine colorectal surgery performed in Hungary in both university and non-university hospitals, and in urban and rural centres.

\section{Acknowledgements}

The authors thank B. Barabás for help with statistical calculations, and R. O'Connell for professional and linguistic advice.

Disclosure. The authors declare no conflict of interest.

\section{References}

1. Devane LA, Proud D, O'Connell PR, Panis Y. A European survey of bowel preparation in colorectal surgery. Colorectal Dis 2017;19: 0402-0406

2. Nelson RL, Gladman E, Barbateskovic M. Antimicrobial prophylaxis for colorectal surgery. Cochrane Database Syst Rev 2014;(5):CD001181

3. Güenaga KF, Matos D, Wille-Jørgensen P. Mechanical bowel preparation for elective colorectal surgery. Cochrane Database Syst Rev 2011;(9):CD001544

4. Gustafsson UO, Scott MJ, Hubner M, Nygren J, Demartines N, Francis $\mathrm{N}$ et al. Guidelines for perioperative care in elective colorectal surgery: Enhanced Recovery After Surgery (ERAS ${ }^{\circledR}$ ) Society recommendations: 2018. World J Surg 2019;43:659-695
5. Nichols RL, Condon RE, Gorbach SL, Nyhus LM. Efficacy of preoperative antimicrobial preparation of the bowel. Ann Surg 1972;176:227-232

6. Althumairi AA, Canner JK, Pawlik TM, Schneider E, Nagarajan N, Safar B et al. Benefits of bowel preparation beyond surgical site infection. Ann Surg 2016;264:1051-1057

7. Moghadamyeghaneh Z, Hanna MH, Carmichael JC, Mills SD, Pigazzi A, Nguyen NT et al. Nationwide analysis of outcomes of bowel preparation in colon surgery. J Am Coll Surg 2015;220: 912-920

8. Tiernan JP, Liska D. Enhanced recovery after surgery: recent developments in colorectal surgery. Surg Clin North Am 2018;98: 1241-1249

9. Migaly J, Bafford AC, Francone TD, Gaertner WB, Eskicioglu C, Bordeianou L et al.The American Society of Colon and Rectal Surgeons Clinical Practice Guidelines for the Use of Bowel Preparation in Elective Colon and Rectal Surgery. Dis Colon Rectum 2019;62:3-8

10. Holubar SD, Hedrick T, Gupta R, Kellum J, Hamilton M, Gan TJ et al. American Society for Enhanced Recovery (ASER) and Perioperative Quality Initiative (POQI) joint consensus statement on prevention of postoperative infection within an enhanced recovery pathway for elective colorectal surgery. Perioper Med 2017;6:4

11. Scarborough JE, Mantyh CR, Sun Z, Migaly J. Combined mechanical and oral antibiotic bowel preparation reduces incisional surgical site infection and anastomotic leak rates after elective colorectal resection: an analysis of colectomy-targeted ACS NSQIP. Ann Surg 2015;262:331-337

12. Morris MS, Graham LA, Chu DI, Cannon JA, Hawn MT. Oral antibiotic bowel preparation significantly reduces surgical site infection rates and readmission rates in elective colorectal surgery. Ann Surg 2015;261:1034-1040

13. Kiran RP, Murray ACA, Chiuzan C, Estrada D, Forde K. Combined preoperative mechanical bowel preparation with oral antibiotics significantly reduces surgical site infection, anastomotic leak, and ileus after colorectal surgery. Ann Surg 2015;262:416-425

14. Vallance A, Wexner S, Berho M, Cahill R, Coleman M, Haboubi N et al. A collaborative review of the current concepts and challenges of anastomotic leaks in colorectal surgery. Colorectal Dis 2017;19:01-O12

15. Chadi SA, Fingerhut A, Berho M, DeMeester SR, Fleshman JW, Hyman $\mathrm{NH}$ et al. Emerging trends in the etiology, prevention, and treatment of gastrointestinal anastomotic leakage. J Gastrointest Surg 2016;20:2035-2051 
16. Toh JWT, Phan K, Hitos K, Pathma-Nathan N, El-Khoury T, Richardson AJ et al. Association of mechanical bowel preparation and oral antibiotics before elective colorectal surgery with surgical site infection: a network meta-analysis. JAMA Netw Open 2018;1:e183226

17. Roos D, Dijksman LM, Tijssen JG, Gouma DJ, Gerhards MF, Oudemans-Van Straaten HM. Systematic review of perioperative selective decontamination of the digestive tract in elective gastrointestinal surgery. BrJ Surg 2013;100:1579-1588

18. Rollins KE, Javanmard-Emamghissi H, Acheson AG, Lobo DN. The role of oral antibiotic preparation in elective colorectal surgery: a meta-analysis. Ann Surg 2019;270:43-58

19. Chen M, Song X, Chen LZ, Lin ZD, Zhang XL. Comparing mechanical bowel preparation with both oral and systemic antibiotics versus mechanical bowel preparation and systemic antibiotics alone for the prevention of surgical site infection after elective colorectal surgery: a meta-analysis of randomized controlled clinical trials. Dis Colon Rectum 2016;59:70-78

20. Gomila A, Carratalà J, Camprubí D, Shaw E, Badia JM, Cruz A et al. Risk factors and outcomes of organ-space surgical site infections after elective colon and rectal surgery. Antimicrob Resist Infect Control 2017;6:40

21. Mik M, Berut M, Trzcinski R, Dziki L, Buczynski J, Dziki A. Preoperative oral antibiotics reduce infections after colorectal cancer surgery. Langenbecks Arch Surg 2016;401:1153-1162

22. Battersby CLF, Hajibandeh S, Hajibandeh S. Oral antibiotics as adjunct to systemic antibiotics and mechanical bowel preparation for prevention of surgical site infections in colorectal surgery. Do we really need more trials? Dis Colon Rectum 2018;61:e341-e342

23. Espin-Basany E, Sanchez-Garcia JL, Lopez-Cano M, LozoyaTrujillo R, Medarde-Ferrer M, Armadans-Gil L et al. Prospective, randomised study on antibiotic prophylaxis in colorectal surgery. Is it really necessary to use oral antibiotics? Int J Colorectal Dis 2005;20:542-546

24. Kobayashi M, Mohri Y, Tonouchi H, Miki C, Nakai K, Kusunoki M; Mie Surgical Infection Research Group. Randomized clinical trial comparing intravenous antimicrobial prophylaxis alone with oral and intravenous antimicrobial prophylaxis for the prevention of a surgical site infection in colorectal cancer surgery. Surg Today 2007;37:383-388

25. Ikeda A, Konishi T, Ueno M, Fukunaga Y, Nagayama S, Fujimoto $Y$ et al. Randomized clinical trial of oral and intravenous versus intravenous antibiotic prophylaxis for laparoscopic colorectal resection. Br J Surg 2016;103:1608-1615
26. Uchino M, Ikeuchi H, Bando T, Chohno T, Sasaki H, Horio Y et al. Efficacy of preoperative oral antibiotic prophylaxis for the prevention of surgical site infections in patients with Crohn disease. Ann Surg 2019;269:420-426

27. Goto S, Hasegawa S, Hata H, Yamaguchi T, Hida K, Nishitai R et al. Differences in surgical site infection between laparoscopic colon and rectal surgeries: sub-analysis of a multicenter randomized controlled trial (Japan-Multinational Trial Organization PREV 07-01). Int J Colorectal Dis 2016;31:1775-1784

28. Anjum N, Ren J, Wang G, Li G, Wu X, Dong H et al. A randomized control trial of preoperative oral antibiotics as adjunct therapy to systemic antibiotics for preventing surgical site infection in clean contaminated, contaminated, and dirty type of colorectal surgeries. Dis Colon Rectum 2017;60:1291-1298

29. McSorley ST, Steele CW, McMahon AJ. Meta-analysis of oral antibiotics, in combination with preoperative intravenous antibiotics and mechanical bowel preparation the day before surgery, compared with intravenous antibiotics and mechanical bowel preparation alone to reduce surgical-site infections in elective colorectal surgery. BJS Open 2018;2:185-194

30. Glasbey JC, Blanco-Colino R, Kelly M, Singh B, Nepogodiev D, Bhangu $\mathrm{A}$ et al. Association of mechanical bowel preparation with oral antibiotics and anastomotic leak following left sided colorectal resection: an international, multi-centre, prospective audit. Colorectal Dis 2018;20:15-32.

31. Koskenvuo L, Lehtonen T, Koskensalo S, Rasilainen S, Klintrup $\mathrm{K}$, Ehrlich A, et al. Mechanical and oral antibiotic bowel preparation versus no bowel preparation for elective colectomy (MOBILE): a multicentre, randomised, parallel, single-blinded trial. Lancet (London, England) 2019;394:840-848

32. https://www.clinicaltrials.gov/ct2/show/NCT03856671?term $=0$ ral+antibiotic+prophylaxis\&cond $=$ Colorectal + Carcinoma\& draw $=1$ \&rank $=7$ (accessed 1 February 2020)

33. https://eudract.ema.europa.eu/ctr-search/search?query=oral+ antibiotic+prophylaxis+AND+colorectal (accessed 1 February 2020)

34. Berríos-Torres SI, Umscheid CA, Bratzler DW, Leas B, Stone EC, Kelz RR et al. Centers for Disease Control and Prevention guideline for the prevention of surgical site infection, 2017. JAMA Surg 2017;152:784-791

35. Lai EJ, Calderwood AH, Doros G, Fix OK, Jacobson BC. The Boston bowel preparation scale: a valid and reliable instrument for colonoscopy-oriented research. Gastrointest Endosc 2009;69: $620-625$ 